\title{
JCOG trials of systemic chemotherapy for unresectable or recurrent gastric cancer
}

\author{
NARIKAZU BOKU \\ Division of Gastrointestinal Oncology, Shizuoka Cancer Center, 1007 Shimonagakubo, Nagaizumi-cho, Shizuoka 411-8777, Japan
}

\begin{abstract}
From the late 1980s to the early 1990s, the Gastrointestinal Oncology Study Group of the Japan Clinical Oncology Group (GIOSG/JCOG) conducted several phase II studies, some of which evaluated oral fluoropyrimidines and others of which introduced Western regimens to Japanese patients. Thereafter, in the phase III study JOCG9205 comparing 5fluorouracil (5-FU), 5-FU plus cisplatin (CDDP) (FP), and uracil and tegafur (UFT) plus mitomycin (UFTM), neither FP nor UFTM showed a survival benefit over 5-FU alone. Whereas irinotecan (CPT-11) and S-1 (new oral fluoropyrimidine) were developed with promising action against gastric cancer in the late 1990 s, these agents cannot be used for patients with impaired oral intake and bowel passage caused by severe peritoneal metastasis. Sequential methotrexate (MTX) and 5-FU (MF) therapy showed substantial action against peritoneal metastasis. Thus, GIOSG/JCOG followed different treatment strategies according to the presence or absence of severe peritoneal metastasis. The phase III study JCOG9912, comparing 5-FU, CPT-11 plus CDDP, and $S-1$, showed a highly significant noninferiority of $S-1$ to 5-FU in overall survival associated with acceptable toxicities and concluded that $\mathrm{S}-\mathbf{1}$ should be considered for the standard chemotherapy for gastric cancer without severe peritoneal metastasis. For patients with severe peritoneal metastasis, the phase III study JCOG0106 compares MF to 5-FU. In that study, patient enrollment has been completed and a final analysis is planned at the end of 2008 . The randomized phase II study JCOG0407 compares the best available 5-FU with weekly paclitaxel after failure in first-line chemotherapy containing 5-FU.
\end{abstract}

Key words Gastric cancer $\cdot \mathrm{JCOG} \cdot$ Fluoropyrimidine $\cdot$ Cisplatin $\cdot$ Irinotecan

Offprint requests to: $\mathrm{N}$. Boku

Received: March 31, 2008 / Accepted: May 5, 2008

\section{Introduction}

Gastric cancer is the second leading cause of cancerrelated death worldwide, accounting for more than 20 deaths per 100000 population annually in East Asia, Eastern Europe, and parts of Central and South America [1]. Gastric cancer is also the second most frequent cause of cancer death after lung cancer in Japan (despite the markedly higher curability obtained by early detection and surgery than in Western countries), because unresectable or recurrent gastric cancer shows a poor prognosis. The development of effective standard chemotherapy is warranted.

For unresectable or recurrent gastric cancer, several phase III trials have demonstrated that a 5-fluorouracil (5-FU)-based regimen provides a survival benefit to these patients over best supportive care [2-4]. Although quite a few randomized trials [5-12], using anthracyclin, mitomycin C (MMC), 5-FU, methotrexate (MTX), and cisplatin (CDDP), were carried out before the early 1990s, none of the chemotherapy regimens showed a survival benefit over 5-FU alone, and no worldwide consensus about a standard regimen has been obtained. For two decades, the Gastrointestinal Oncology Study Group of the Japan Clinical Oncology Group (GIOSG/ JCOG) has conducted several clinical trials to establish standard chemotherapy (Table 1).

\section{GIOSG/JCOG trials from establishment to JCOG $9205[13,15,16]$}

From the late 1980 s to the early 1990 s, all of the GIOSG/ JCOG trials were phase II studies. During this period some oral fluoropyrimidines were developed in Japan, among which tegafur (FT) and FT plus uracil (UFT) were the most popular. Because the response rates of monotherapy with fluoropyrimidines were not satisfactory, combination chemotherapy with these agents was 
Table 1. GIOSG/JCOG clinical trials for gastric cancer

\begin{tabular}{|c|c|c|}
\hline $\begin{array}{l}\text { Study } \\
\text { number }\end{array}$ & Phase & Study \\
\hline $8501[13]$ & rII & A cooperative randomized study of tegafur plus mitomycin $\mathrm{C}$ in the treatment of advanced gastric cancer \\
\hline $8903[14]$ & II & A phase II study of EAP (etoposide, adriamycin, and cisplatin) in patients with advanced gastric cancer \\
\hline 9001 [15] & II & Phase II study of protracted infusional 5-fluorouracil combined with cisplatin for advanced gastric cancer \\
\hline $9205[16]$ & III & $\begin{array}{l}\text { Randomized phase III trial of fluorouracil alone versus fluorouracil plus cisplatin versus uracil and } \\
\text { tegafur plus mitomycin C in patients with unresectable advanced gastric cancer }\end{array}$ \\
\hline $9207[28]$ & II & $\begin{array}{l}\text { A phase II study of sequential methotrexate and 5-fluorouracil chemotherapy in patients with previously } \\
\text { treated gastric cancer }\end{array}$ \\
\hline 9410 [29] & II & A phase II study of doxifluridine in elderly patients with advanced gastric cancer \\
\hline $9603[23]$ & II & $\begin{array}{l}\text { A phase II study of sequential methotrexate and 5-fluorouracil for advanced gastric cancer with } \\
\text { malignant ascites }\end{array}$ \\
\hline $9912[20]$ & III & $\begin{array}{l}\text { Randomized phase III study of 5-fluorouracil (5-FU) alone versus combination of irinotecan and } \\
\text { cisplatin (CP) versus S-1 alone in advanced gastric cancer }\end{array}$ \\
\hline 0106 & III & $\begin{array}{l}\text { Randomized phase III study of 5-FU continuous infusion (5-FUci) versus MTX + 5-FU sequential } \\
\text { therapy (MF) in gastric cancer with peritoneal metastasis }\end{array}$ \\
\hline 0407 & rII & $\begin{array}{l}\text { Randomized phase II study of best available 5-FU versus low-dose paclitaxel in gastric cancer with } \\
\text { peritoneal metastasis refractory to 5-FU containing regimens }\end{array}$ \\
\hline
\end{tabular}

investigated. In a randomized phase II study (JCOG 8501) comparing FT plus MMC (FTM) with UFT plus MMC (UFTM) [13], UFTM showed a higher response rate than FTM. It was concluded that UFTM would be a candidate for a test arm in a future phase III trial.

Subsequently, the GIOSG/JCOG introduced Western chemotherapy regimens such as etoposide plus doxorubicin plus CDDP (EAP) [14] and 5-FU plus CDDP (FP) [15]. Although a high response rate and a 5-year survival of $10 \%$ were obtained in the EAP trial, treatmentrelated deaths occurred in $10 \%$ of the patients. While the dose and schedule of FP in Japan were slightly modified from those in Western trials [12], the FP trial [15] showed a response rate and survival similar to those of Western trials. Therefore, the FP regimen was selected for a future phase III trial.

When the GIOSG/JCOG was planning a randomized phase III trial (JCOG 9205) [16], it was reported that, in a Western phase III trial [9], combination chemotherapy consisting of 5-FU, doxorubicin, and $\mathrm{MMC}$ (FAM) did not show a survival benefit over 5-FU alone Therefore, the GIOSG/JCOG decided to adopt 5-FU alone for the control arm in that trial, which was conducted as a three-arm phase III trial comparing FP and UFTM with 5-FU alone. FP did not show significantly longer survival despite its higher response rate and longer progression-free survival; this was associated with more severe toxicities than 5-FU alone. Furthermore, UFTM resulted in the worst survival among the three treatment arms and showed more severe toxicities than 5-FU alone. JCOG 9205 concluded that 5-FU alone would remain as the control arm for a subsequent phase III study. Although no survival benefit of combination chemotherapy containing 5-FU and CDDP over 5 -FU alone has been confirmed in several phase III trials $[8,9,12]$, the FP regimen has been the one most widely used for unresectable and recurrent gastric cancer all over the world.

\section{JCOG 9912}

In the late 1990s, some new antitumor agents were developed for gastric cancer in Japan. Combination chemotherapy of irinotecan (CPT-11) plus CDDP showed a response rate of $59 \%$ and a median survival time of 322 days, associated with grade 4 neutropenia (57\%), and grade 3 or 4 diarrhea (20\%) [17]. S-1, a new oral fluoropyrimidine, consisting of FT, 5-chloro-2, 4dihydropyrimidine, and potassium oxonate, showed a response rate of $45 \%$ and a high 2 -year survival rate of $17 \%$ in a total of 100 patients in two phase II studies, associated with low incidences of grade 3 or 4 toxicities[18, 19]. These results seemed very promising, and both a combination of CPT-11 plus CDDP and monotherapy with S-1 were adopted for test arms. The GIOSG/JCOG then planned a three-arm phase III study (JCOG 9912) to investigate the superiority of CPT-11 plus CDDP and the non-inferiority of S-1 compared to continuous infusion of 5-FU [20] (Fig. 1). The primary endpoint was overall survival, and secondary endpoints were time to treatment failure, nonhospitalized survival, adverse events, and response rate.

While the eligibility criteria of JCOG 9912 were similar to those of other recent phase III studies, the specific points of JCOG 9912 were that the presence of measurable lesions according to Response Evaluation Criteria in Solid Tumors (RECIST) was not mandatory and that patients with severe peritoneal metastasis were 


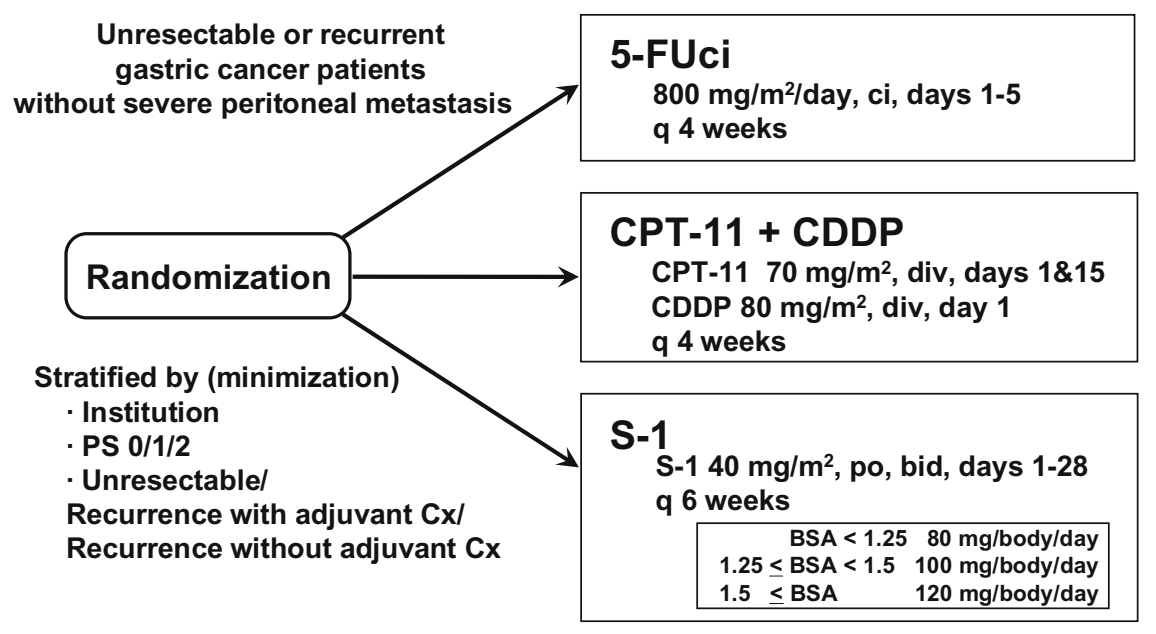

Fig. 1. Schema of JCOG 9912 [20]. 5-FU, 5 fluorouracil; $c i$, continuous infusion; CPT-11 irinotecan; $C D D P$, cisplatin; div, $P S$, performance status; $C x$, chemotherapy; $B S A$, body surface area

Table 2. Incidence (\%) of grade 3 or greater adverse events in JCOG 9912 [20]

\begin{tabular}{|c|c|c|c|}
\hline Regimen & $\begin{array}{c}5-\mathrm{FU} \\
(n=234)\end{array}$ & $\begin{array}{c}\text { CPT-11 + CDDP } \\
(n=236)\end{array}$ & $\begin{array}{c}\mathrm{S}-1 \\
(n=234)\end{array}$ \\
\hline Leukocytes & 0 & 41.5 & 0.9 \\
\hline Neutrophils & 1.3 & 65.0 & 5.6 \\
\hline Hemoglobin & 15.5 & 39.3 & 12.8 \\
\hline Febrile neutropenia & 0 & 9.4 & 0 \\
\hline Infection with grade 3 or 4 neutropenia & 0 & 7.7 & 0.4 \\
\hline Infection without neutropenia & 3.9 & 3.8 & 5.6 \\
\hline AST & 4.7 & 2.6 & 4.7 \\
\hline ALT & 3.4 & 2.6 & 3.4 \\
\hline Bilirubin & 3.0 & 1.3 & 4.3 \\
\hline Creatinine & 0 & 2.1 & 0.9 \\
\hline Hyponatremia & 6.5 & 22.6 & 5.2 \\
\hline Fatigue & 1.7 & 10.3 & 5.1 \\
\hline Anorexia & 12.5 & 32.9 & 12.4 \\
\hline Diarrhea & 0.4 & 9.0 & 7.7 \\
\hline Nausea & 6.9 & 20.5 & 5.6 \\
\hline Stomatitis & 3.0 & 0 & 1.7 \\
\hline Treatment-related death ${ }^{\mathrm{a}}$ & 0 & 1.3 & 0.4 \\
\hline
\end{tabular}

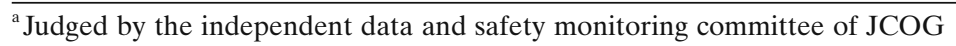

excluded. The treatment schedules were: continuous infusion of 5 -FU $\left(800 \mathrm{mg} / \mathrm{m}^{2}\right.$ per day $)$ for 5 days, repeated every 4 weeks, in the 5-FU arm; administration of both CPT-11 $\left(70 \mathrm{mg} / \mathrm{m}^{2}\right)$ and (CDDP $\left.80 \mathrm{mg} / \mathrm{m}^{2}\right)$ on day 1 and additional CPT-11 on day 15 , repeated every 4 weeks, in the CPT-11 plus CDDP arm; and oral administration of $\mathrm{S}-1$ ( $40 \mathrm{mg} / \mathrm{m}^{2}$, b.i.d.) for 4 weeks, followed by 2 weeks' rest, repeated every 6 weeks, in the S-1 arm. Actually, 704 patients were accrued for 5 years. Final analysis was carried out in February 2007, 1 year after the last patient enrollment.

Table 2 summarizes the toxicities in JCOG 9912. Leucopenia and neutropenia were the most severe toxicities, and grade 3 or 4 hyponatremia, fatigue, anorexia, diarrhea, and nausea were most frequently observed in the CPT-11 plus CDDP arm. Only diarrhea was more severe in the S-1 arm than in the 5-FU arm, while there were no remarkable differences in the incidences of other toxicities between the S-1 arm and the 5-FU arm. It was considered that monotherapy with S-1 was feasible.

Table 3 summarizes the results for the efficacy of JCOG 9912. The response rate of the CPT-11 plus CDDP arm was $38 \%$, and those of the S-1 and 5-FU arms were $28 \%$ and $9 \%$. The median progression-free survival time of the 5-FU arm was 2.9 months; that of the CPT-11 plus CDDP arm, 4.8 months; and that of the S-1 arm, 4.2 months. Thus, it can be said that the antitumor effect of CPT-11 plus CDDP was the best among the three treatment arms. The median time to treatment failure of the 5-FU arm was 2.3 months; CPT-11 plus CDDP arm, 3.7 months; and the S-1 arm, 4.0 months. As for the reasons for treatment failure, more than $85 \%$ of the patients stopped treatment due 
Table 3. Efficacy in JCOG 9912 [20]

\begin{tabular}{lccc}
\hline Regimen & $\begin{array}{c}5-\mathrm{FU} \\
(n=234)\end{array}$ & $\begin{array}{c}\text { CPT-11+CDDP } \\
(n=236)\end{array}$ & $\begin{array}{c}\mathrm{S}-1 \\
(n=234)\end{array}$ \\
\hline Response rate by RECIST $(\%)$ & $9(15 / 175)$ & $38(68 / 181)$ & $28(49 / 175)$ \\
PFS (months) & 2.9 & 4.8 & 4.2 \\
& - & $P<0.001^{*}$ & $P=0.001^{*}$ \\
TTF (months) & 2.3 & 3.7 & 4.0 \\
& - & $P=0.014^{*}$ & $P<0.001^{*}$ \\
MST (months) & 10.8 & 12.3 & 11.4 \\
& - & $P=0.055^{*}$ & $P=0.034^{*}$ \\
& & & $P<0.001^{* *}$ \\
\hline
\end{tabular}

$P$ compared with 5-FU: $* P$ value for superiority; $* * P$ value for non-inferiority

PFS, progression-free survival; TTF, time to treatment failure

Table 4. Recent phase III trials in Japan

\begin{tabular}{|c|c|c|c|c|c|}
\hline Trial & Regimen & $n$ & RR (\%) & PFS (months) & MST (months) \\
\hline \multirow[t]{3}{*}{ JCOG 9912 [20] } & $5-\mathrm{FU}$ & 234 & 9 & 2.9 & 10.8 \\
\hline & CPT-11+CDDP & 236 & 38 & 4.8 & 12.3 \\
\hline & S-1 & 234 & 28 & 4.2 & 11.4 \\
\hline \multirow[t]{2}{*}{ SPIRITS [21] } & S-1 & 150 & 31 & 4.0 & 11.0 \\
\hline & $\mathrm{S}-1+\mathrm{CDDP}$ & 148 & 54 & 6.0 & 13.0 \\
\hline \multirow{2}{*}{$\begin{array}{l}\text { GC 0301/TOP } 002 \\
{[22]}\end{array}$} & S-1 & 160 & 27 & - & 10.5 \\
\hline & S-1 + CPT-11 & 155 & 42 & - & 12.8 \\
\hline
\end{tabular}

to disease progression in the 5-FU arm and S-1 arm, while more than $30 \%$ of the patients stopped treatment for reasons related to toxicities in the CPT-11 plus CDDP arm. Substantial toxicities seemed to shorten the time to treatment failure in the CPT-11 plus CDDP arm.

As for the overall survival, the CPT-11 plus CDDP arm showed the best survival until 1 year, and the median survival times (MSTs) of the 5-FU, CPT-11 plus CDDP, and S-1 arms were 10.8, 12.3, and 11.4 months, while the S-1 arm showed the best survival after 1 year. As a whole, the hazard ratio to 5-FU of CPT-11 plus CDDP was 0.85 (95\% confidence interval [CI], $0.70-1.04 ; P=0.055)$ and that of S-1 was $0.83(95 \% \mathrm{CI}$, $0.68-1.01 ; P=0.034$ for superiority, $P<0.001$ for noninferiority). Because the significance level for superiority after confirming the non-inferiority of S-1 was pre-specified to be 0.025 , it was concluded that only the non-inferiority of S-1 was shown to be statistically significant, while there was no consensus about the significance level, 0.025 or 0.05 , for superiority after confirming non-inferiority.

Because infusion chemotherapy is commonly performed with hospitalization in Japan, it is considered that the non-hospitalized survival reflects a patient's benefit from the quality-of-life point of view. Both the CPT-11 plus CDDP and S-1 arms showed longer nonhospitalized survival compared to the 5-FU arm.

S-1 showed a higher response rate, longer progression-free survival, and longer non-hospitalized survival than 5-FU, associated with feasible toxicities. It can be said that S-1 may be superior to 5-FU in practice. In conclusion, S-1 should be considered as standard chemotherapy for unresectable or recurrent gastric cancer.

\section{Other phase III trials}

There have been two other randomized phase III trials with $\mathrm{S}-1$ as a control arm. One investigated the superiority of S-1 plus CDDP compared with S-1 alone in regard to overall survival S-1 plus cisplatin vs $\mathrm{S}-1$ in RCT in the treatment for stomach cancer (SPIRITS trial) [21]. Overall survival was significantly longer in the S-1 plus CDDP arm (MST, 13.0 months) than in the S-1 arm (MST, 11.0 months; $P=0.04$ ). The other phase III trial compared the combination of S-1 plus CPT-11 with S-1 (GC 0301/TOP 002) [22]. Although the MST of the S-1 arm was 10.5 months and that of the S-1 plus CPT-11 arm was 12.8 months, S- 1 plus CPT-11 did not show significant superiority $(P=0.23)$. The treatment results for the S-1 arm, in terms of response rates and overall survival, were very similar in the three phase III trials (Table 4). Generally, it is recognized that S-1 plus CDDP is the standard treatment for unresectable or recurrent gastric cancer in Japan. Of note, there are two global trials investigating the non-inferiority of oxaliplatin and/or oral pyrimidine (capecitabine) compared to CDDP and continuous infusion of 5-FU; both of these 
trials have met their primary endpoints. It can be said that Japan and Western countries share a consensus on standard chemotherapy with oral fluoropyrimidine plus platinum for advanced gastric cancer.

However, strictly speaking, none of the combination chemotherapies with oral fluoropyrimidine and platinum have shown superiority to FP, while S-1 monotherapy showed a response rate and progression-free survival similar to those of FP in the JCOG 9205 trial [16] as results of first-line chemotherapy. Of note, because the final analysis of JCOG 9912 [20] was performed just 1 year after the last patient enrollment, approximately $15 \%$ of the patients were censored in the survival curves after 1 year. Therefore, the long-term results of JCOG 9912 have not been obtained yet. While there was a remarkable difference in long-term survival between the arms in the SPIRITS trial (2-year survival rate: $\mathrm{S}-1,15.3 \%$; S-1 + CDDP, $23.6 \%$ ), the 2-year survival rate of the S-1 arm in JCOG 9912 is speculated to be more than $20 \%$ from the survival curve at the final analysis. Therefore, it might be considered that it has not yet been clarified which is the better treatment strategy, i.e., whether monotherapy with S-1 followed by subsequent chemotherapy, or intensive combination chemotherapy such as S-1 plus CDDP as the first-line treatment will have a greater impact on long-term survival. The superiority of S-1 plus CDDP combination chemotherapy to FP is now under investigation by the First-Line Advanced Gastric Cancer Study (FLAGS) trial to confirm whether S-1 plus CDDP can be a standard chemotherapy all over the world.

\section{JCOG 0106}

The peritoneum, as well as liver and lymph nodes, is a major common metastatic site. The incidence of peritoneal metastasis in patients with unresectable or recurrent gastric cancer is higher than $50 \%$. The prognosis of patients with severe peritoneal metastasis is considered to be poor, because it causes various kinds of complications such as ascites, bowel obstruction, and hydronephrosis, causing deterioration of the patient's general condition; also, these patients usually do not have target lesions according to RECIST. For these reasons, patients with severe peritoneal metastasis are generally excluded from clinical trials. Moreover, new drugs such as CPT11 and S-1 cannot be used for these patients; the impaired bowel passage caused by severe peritoneal metastasis causes severe CPT-11 toxicities through the reabsorption of its active metabolite $\mathrm{SN}-38$, and the impairment also prevents the oral administration of S-1. These patients were also excluded from the recent Japanese phase III trials. Therefore, evidence from clinical trials cannot be applied to these patients, and it is considered that a standard chemotherapy for gastric cancer patients with severe peritoneal metastasis has not been established.

The GIOSG/JCOG has decided to use different treatment strategies according to the presence or absence of severe peritoneal metastasis, and planned a phase III trial (JCOG 0106) targeting patients with severe peritoneal metastasis (Fig. 2). In this trial, 5-FU alone was adopted as the control arm, from the results of JCOG 9205, because it was the least toxic and could be applied safely to patients with severe peritoneal metastasis. Sequential therapy of 5-FU and MTX with leucovorin rescue (MTX/5-FU) was adopted as the test arm in this trial, based on the results of a phase II study of this regimen (JCOG 9603 [23]), targeting severe peritoneal metastasis; massive ascites was markedly decreased in 13 out of 37 patients (35\%). The MTX/5-FU regimen is based on biochemical modulation; it is reported that an increase of phosphoribosyl pyrophosphate in cancer cells through the inhibition of purine synthesis by MTX enhances the effects of 5-FU. The major toxicities with this sequential therapy in the JCOG 9603 trial were leucopenia, anemia,

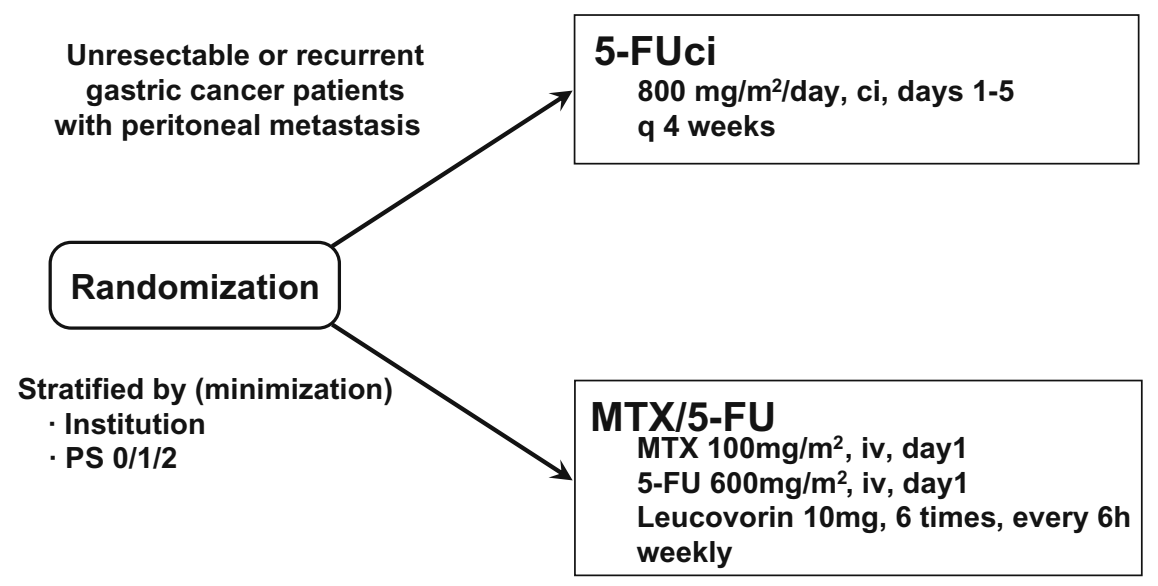

Fig. 2. Schema of JCOG 0106. MTX, methotrexate 


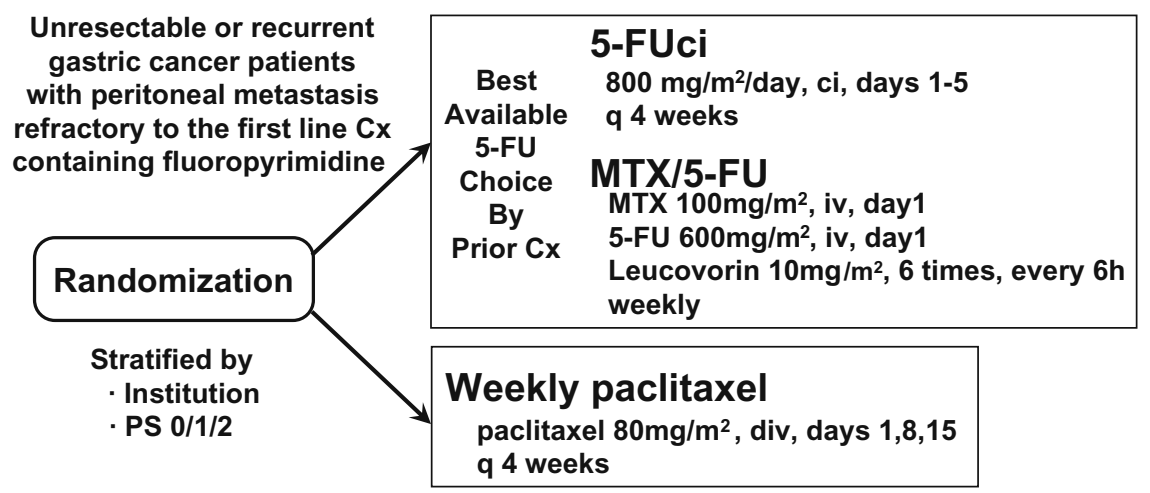

Fig. 3. Schema of JCOG 0407 thrombocytopenia, diarrhea, and renal dysfunction. A total of 237 patients were randomized to either the 5FU arm or the MTX/5-FU arm between October 2002 and April 2007, and the final analysis is planned for the autumn of 2008 .

\section{JCOG 0407}

The survival benefit of second-line chemotherapy for unresectable or recurrent gastric cancer has not been confirmed by randomized phase III trials. In a comparison of the overall survival of patients receiving 5-FU as first-line treatment between the JCOG 9205 trial [16] and the JCOG 9912 trial [20], the MST in the JCOG 9912 trial was markedly longer than that in the JCOG 9205 trial. Between the time of the JCOG 9205 trial and the JCOG 9912 trial, new drugs such as CPT-11, paclitaxel [24], and docetaxel [25] were approved in Japan, and they were widely used as second-line treatment in clinical practice [26, 27]. While around half of the patients in the JCOG 9205 trial received second-line chemotherapy, more than $70 \%$ of the patients in the JCOG 9912 trial received this treatment. It is considered that second-line chemotherapy may contribute to prolongation of survival. At present, several randomized phase III trials of second-line treatment after the failure of first-line treatment with fluoropyrimidine and/or platinum are underway in Japan.

Although not a few patients are complicated with severe peritoneal metastasis after the failure of first-line chemotherapy, second-line chemotherapy is limited because of the patients' poor condition. Because a standard second-line chemotherapy for severe peritoneal metastasis has not been established, it is difficult to decide on the control arm for a randomized trial. From our experience in clinical practice, we believe there are two candidates for treatment arms in such clinical trials. One is the "best available fluoropyrimidine", which is based on a mechanism of cytotoxicity that is different from that of 5-FU; this is achieved through the use of different administration methods from the initial therapy; such as continuous infusion (oral agent) and bolus infusion, with and without biochemical modulation by leucovorin or MTX. The other candidate treatment arm is the weekly administration of paclitaxel, which showed a response rate of around $20 \%$, with fewer toxicities than for tri-weekly administration, even as second-line treatment; also with weekly treatment, a high concentration was maintained in ascites. Thus, the GIOSG/JCOG has started a randomized phase II trial (Fig. 3) in patients with severe peritoneal metastasis, comparing best available 5-FU with weekly administration of paclitaxel as second-line chemotherapy after the failure of first-line chemotherapy containing fluoropyrimidine, and patient accrual is under way.

\section{Conclusion}

In this article, GIOSG/JCOG clinical trials have been reviewed. There have been many phase III clinical trials in Japan and all over the world. The progress of chemotherapy for unresectable and recurrent gastric has been remarkable since the late 1990s, and standard chemotherapy has been established. In the near future, revolutionary progress can be expected through the development of new drugs, including molecular targeting agents.

Acknowledgments JCOG trials were supported mainly by Grants-in-Aid for Cancer Research (11S-3, 11S-4, 14S-3, 14S-4, 17S-3, 17S-5), and Health and Labour Sciences Research Grants for Clinical Cancer Research (H17-Gan-008), from the Ministry of Health, Labour and Welfare of Japan.

\section{References}

1. Parkin DM, Bray F, Ferlay J, Pisani P. Global cancer statistics, 2002. CA Cancer J Clin 2005;55:74-108. 
2. Murad AM, Santiago FF, Petroianu A, Rocha PR, Rodrigues MA, Rausch M. Modified therapy with 5-fluorouracil, doxorubicin, and methotrexate in advanced gastric cancer. Cancer 1993;72: 37-41.

3. Glimelius B, Hoffmann K, Haglund U, Nyren O, Sjoden PO. Initial or delayed chemotherapy with best supportive care in advanced gastric cancer. Ann Oncol 1994;5:189-90.

4. Pyrhonen S, Kuitumen T, Nyandoto P, Kouri M. Randomized comparison of fluorouracil, epidoxorubicin and methotrexate (FEMTX) plus best supportive care alone in patients with nonresectable gastric cancer. Br J Cancer 1995;71:587-91.

5. Cullinan SA, Moertel CG, Fleming TR, Krook JE, Everson LK, Windschitl HE, et al. A comparison of three chemotherapeutic regimens in the treatment of advanced pancreatic and gastric carcinoma. Fluorouracil versus fluorouracil and doxorubicin versus fluorouracil, doxorubicin, and mitomycin. JAMA 1985; 253:2061-7.

6. Wils JA, Klein HO, Wagener DJ, Bleiberg H, Reis H, Korsten F, et al. Sequential high-dose methotrexate and fluorouracil, combined with doxorubicin: a step ahead in the treatment of gastric cancer: a trial of the European Organization for Research and Treatment of Cancer Gastrointestinal Tract Co-operative Group. J Clin Oncol 1991;9:827-31.

7. Kelsen D, Atiq OT, Saltz L, Niedzwiecki D, Ginn D, Chapman D, et al. FAMTX versus etoposide, doxorubicin and cisplatin: a randomized trial in gastric cancer. J Clin Oncol 1992;10:541-8.

8. Kim NK, Park YS, Heo DS, Suh C, Kim SY, Park KC, et al. A phase III randomized study of 5-fluorouracil and cisplatin versus 5-fluorouracil, doxorubicin, and mitomycin $\mathrm{C}$ versus 5-fluorouracil alone in the treatment of advanced gastric cancer. Cancer 1993;71:3813-8.

9. Cullinan SA, Moertel CG, Wieand HS, O'Connel MJ, Poon MA, Krook JE, et al. Controlled evaluation of three drug combination regimen versus fluorouracil alone in the therapy of advanced gastric cancer. J Clin Oncol 1994;12:412-6.

10. Cocconi G, Bella M, Zironi S, Algeri R, Di Costanzo F, De Lisi $\mathrm{V}$, et al. Fluorouracil, doxorubicin, and mitomycin combination versus PELF chemotherapy in advanced gastric cancer: a prospective randomized trial of the Italian Oncology Group for Clinical Research. J Clin Oncol 1994;12:2687-93.

11. Webb A, Cunningham D, Scarffe JH, Harper P, Norman A, Joffe JK, et al. Randomized trial comparing epirubicin, cisplatin, and fluorouracil versus fluorouracil, doxorubicin, and methotrexate in advanced esophagogastric cancer. J Clin Oncol 1997;15: 261-7.

12. Vanhoefer U, Rougier P, Wilke H, Ducreux MP, Lacave AJ, Van Cutsem E, et al. Final results of a randomized phase III trial of sequential high-dose methotrexate, fluorouracil, and doxorubicin versus etoposide, leucovorin, and fluorouracil versus infusional fluorouracil and cisplatin in advanced gastric cancer: a trial of the European Organization for Research and Treatment of Cancer Gastrointestinal Tract Cancer Cooperative Group. J Clin Oncol 2000;18:2648-57.

13. Kurihara M, Izumi T, Yoshida S, Ohkubo T, Suga S. A cooperative randomized study on tegafur plus mitomycin $\mathrm{C}$ in the treatment of advanced gastric cancer. Jpn J Cancer Res 1991;82: 613-20.

14. Shimada Y, Yoshida S, Ohtsu A, Seki S, Saito H. A phase II study of EAP (etoposide, adriamycin and cisplatin) in the patients with advanced gastric cancer: multi-institutional study (abstract). Annual Meeting Japan Society of Clinical Oncology 1991;26: 280 .

15. Ohtsu A, Shimada Y, Yoshida S, Saito H, Seki S, Morise K, et al. Phase II study of protracted infusional 5-fluorouracil combined with cisplatinum for advanced gastric cancer: Report from the
Japan Clinical Oncology Group (JCOG). Eur J Cancer 1994;30A: 2091-3.

16. Ohtsu A, Shimada Y, Shirao K, Boku N, Hyodo I, Saito H, et al. Randomized phase III trial of fluorouracil alone versus fluorouracil plus cisplatin versus uracil and tegafur plus mitomycin in patients with unresectable advanced gastric cancer: the Japan Clinical Oncology Group Study (JCOG9205). J Clin Oncol 2003; 21:54-9.

17. Boku N, Ohtsu A, Shimada Y, Shirao K, Seki S, Saito H, et al. A phase II study of a combination of irinotecan and cisplatin against metastatic gastric cancer. J Clin Oncol 1999;17:319-23.

18. Sakata Y, Ohtsu A, Horikoshi N, Sugimachi K, Mitachi Y, Taguchi T. Late phase II study of novel oral fluoropyrimidine anticancer drug S-1 (1 M tegafur- $0.4 \mathrm{M}$ gimestat- $1 \mathrm{M}$ otastat potassium) in advanced gastric cancer patients. Eur $\mathbf{J}$ Cancer 1998;34:1715-20.

19. Koizumi W, Kurihara M, Nakano S, Hasegawa K. Phase II study of S-1, a novel oral derivative of 5-fluorouracil, in advanced gastric cancer. Oncology 2000;58:191-7.

20. Boku N, Yamamoto S, Shirao K, Doi T, Sawaki A, Koizumi W, et al. Randomized phase III study of 5-fluorouracil (5-FU) alone versus combination of irinotecan and cisplatin (CP) versus S-1 alone in advanced gastric cancer (JCOG9912). Annual meeting of ASCO, 2007; abstract LBA4513.

21. Koizumi W, Narahara H, Hara T, Takagane A, Akiya T, Takagi M, et al. S-1 plus cisplatin versus S-1 alone for first-line treatment of advanced gastric cancer (SPIRITS trial): a phase III trial. Lancet Oncol 2008;9:215-21.

22. Imamura H, Iiishi H, Tsuburaya A, Hatake K, Imamoto H, Esaki $\mathrm{T}$, et al. Randomized phase III study of irinotecan plus S-1 (IRIS) versus S-1 alone as first-line treatment for advanced gastric cancer (GC0301/TOP002). Gastrointestinal Cancers Symposium 2008: abstract 5 .

23. Yamao T, Shimada Y, Shirao K, Ohtsu A, Ikeda N, Hyodo I, et al. A phase II study of sequential methotrexate and 5fluorouracil for advanced gastric cancer with malignant ascites: a report from the Gastrointestinal Oncology Study Group of the Japan Clinical Oncology Group, JCOG 9603 Trial. Jpn J Clin Oncol 2004;34:316-22.

24. Yamada Y, Ohtsu A, Boku N, Hyodo I, Saitoh H, Miyata Y, et al. Phase II trial of paclitaxel by 3-h infusion for advanced gastric cancer with short premedication for prophylaxis against paclitaxel-associated hypersensitivity reactions. Ann Oncol 2001; 12:1133-7.

25. Sulkes A, Smyth J, Sessa C, Dirix LY, Vermoken JB, Kaye S, et al. Docetaxel (Taxotere) in advanced gastric cancer: results of a phase II clinical trial. EORTC Early Clinical Trials Group. Br J Cancer. 1994;70:380-3.

26. Hironaka S, Zenda S, Boku N, Fukutomi A, Yoshino T, Onozawa Y. Weekly paclitaxel as second-line chemotherapy for advanced or recurrent gastric cancer. Gastric Cancer 2006;9:14-8.

27. Ueda S, Hironaka S, Boku N, Fukutomi A, Yoshino T, Onozawa Y. Combination chemotherapy with irinotecan and cisplatin in pretreated patients with unresectable or recurrent gastric cancer. Gastric Cancer 2006;9:203-7.

28. Hamaguchi T, Shirao K, Yamamichi N, Hyodo I, Koizumi W, Seki S, et al. A phase II study of sequential methotrexate and 5fluorouracil chemotherapy in previously treated gastric cancer: a report from the Gastrointestinal Oncology Group of the Japan Clinical Oncology Group, JCOG 9207 Trial. Jpn J Clin Oncol 2008;38:432-7.

29. Ikeda N, Shimada Y, Ohtsu A, Boku N, Tsuji Y, Saito H, et al. A phase II study of doxifluridine in elderly patients with advanced gastric cancer: Japan Clinical Oncology Group Study (JCOG9410). Jpn J Clin Oncol 2002;32:90-4. 